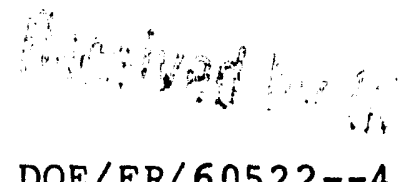

$\mathrm{DOE} / \mathrm{ER} / 60522--4$

DE91 005130

\title{
DEVELOPMENT AND APPLICATION OF PHOTOSENSITIVE DEVICE SYSTEMS TO STUDIES OF BIOLOGICAL AND ORGANIC MATERIALS
}

\author{
First Year Progress Report \\ For the Period Jal.uary 1, 1990 - Decembe: 31, 1990 \\ Sol M. Gruner \& Geo. T. Reynolds \\ Joseph Henry Laboratoris: Dept. of Physics \\ Princeton University \\ Princeton, New Jersey 08544
}

July 12,1990

Prepared for

THE U.S. DEPARTMENT OF ENERGY

GRANT NO. DE-FG-02-87ER60522

\section{MASTER}

\section{NOTICE}

This report was prepared as an account of work sponsored by the United States Government. Neither the United States nor the Department of Energy, nor any of their employees, nor any of their contractors, subcontractors, or their employees, makes any warranty, express or implied, or assumes any legal liability of responsibility for the accuracy, completeness, and usefulness of any information, apparatus, product or process disclosed or represents that its use would not infringe privately-owned rights.

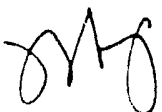


I) INTRODUCTION \& SUMMARY

This report describes the progress of the first year of a 3-year DOE grant DE-FG-0287 ER60522 for the fiscal period $1 / 1 / 90$ to $12 / 31 / 90$, as of July, 1990 . The overall goals of the grant are to develop novel instrumentation and techniques for the performance of biological and materials research and to apply the new developments to basic biological and materials research problems. Since the last progress report, dated June, 1989, there has been significant progress on most of the origionally proposed instrumentation and applications research. The research proposed for the next year has not changed from that origionally listed in the 3-year proposal.

The research is well guided by the philosophy that useful instrumentation development and basic biological research are inseparably coupled and proceed most effectively when the instrumentation development is driven by specific needs of basic research problems. To insure this coupling, the Princeton Biophysics group has avoided the division of the group into "instrumentation" and "applications" specialists; rather, each group member is simultaneously involved in both the development of the instrumentation and the application to basic research problems. However, the progress of the work is most logically reported by separately describing instrumentation and basic research developments. Please bear in mind, however, that, in practice, the basic research is made possible by the novel instrumentation and that the relevance of the instrumentation development follows from the demands of the basic research.

In summary, specific instrumentation accomplishments during the last year include:

- An experimental large area CCD x-ray detector was fabricated and subjected to synchrotron radiation applications testing;

- There has been good progress in the assembly of a novel fiber-optically coupled CCD $x$-ray detector; 
- New phosphor screens for diffraction applications were developed;

- X-ray detector software was developed and applied;

- High pressure $\mathrm{x}$-ray apparatus was designed, built, and tested; and

- Studies on the feasibility of lyoluminescent dosimetry were performed.

Specific basic research accomplishments include:

* New x-ray structure determination methods were developed and applied to biomembrane lipid phases;

* A novel mechanism for general anesthesia was proposed;

* The elastic properties of membranes were investigated, both theoretically and experimentally;

* The effects of high pressures on membranes were studied;

* Neutron diffraction was used to probe mesophase structure; and

* Novel lipid and surfactant systems were characterized.

Each of these accomplishments are detailed, below.

\section{II) INSTRUIMENTATION ACCOMPLISHMENTS}

\section{A) Fabrication and Testing of a Large-Area CCD X-ray Deiector}

CCD-based detector systems promise to revolutionize $x$-ray technology, especially at synchrotron sources. One of the most challenging, and certainly one of the most promising, biological diffraction problems is the collection of time-resolved Laue protein diffraction data. The bottleneck in the utilization of the Laue technique is the development of $x$-ray detectors capable of recording thousands of spots simultaneously, at many wavelengths, with very high spatial resolution and dynamic range. Very large area CCDs are the only known detector technology which seems suitable when small spatial features are involved, as is the case with microcrystals. 
Toward this end, and in collaboration with Princeton Scientific Instruments (Princeton, NJ), a CCD detector was assembled which utilized an experimental TEK $2048 \times 2048$ pixel CCD. This is the largest CCD which has ever been manufactured and is easily the largest to have been applied to diffraction problems. The CCD was directly coupled to a $4 \mathrm{~mm}$ thick fiber-optic plate which had an overlaid phosphor. The chip area was sufficiently large ( $3025 \mathrm{sq} . \mathrm{mm})$ that no reduction of the $\mathrm{x}$-ray irnage was required; this allowed direct coupling without the use of an intervening image intensifier while still maintaining quantum limited operation (Deckman \& Gruner, 1986). Fabrication of the detector required the development of a liquid-based method of coupling the CCD to the fiber optic plate which was capable of surviving repeated cooling cycles to the $-45^{\circ} \mathrm{C}$ detector operating temperature. New phosphor fabrication procedures were also required to maintain adequate efficiency and spatial resolution. The resultant prototype device was characterized as having a spatial resolution limited by the CCD pixel pitch (27 um), a dynamic range of roughly 5000 , and a detective quantum efficiency of nearly unity.

The detector was applied to a test run at the CHESS synchrotron source at Cornell University, in collaboration with Dr. D. Bilderback of the CHESS staff, at the end of June, 1990. Laue data was collected on protein crystals and on gallium arsenide as well as monochromatic data on other samples. The detector performed superbly. Less data was collected than hoped, but this was entirely due to problems with the synchrotron source and with beam-line components provided by the CHESS staff. The data is currently being analyzed and will be compared to data collected by the existing image plate detector system at use at CHESS. It is already clear that the resolution of the detector allowed the discovery of beam collimation problems which were not apparent with the crude spatial resolution of the image plate system. Another synchrotron run may be performed, depending on the 
outcome of the data analysis. In either case, adequate data was collected to evaluate the feasibility of the detector concept.

Initial results were presented as an invited keynote address on CCD detectors by Dr. Mark Tate, at the Third International Conference on Biophysics and Synchrotron Radiation (July 2-6, 1990, Stanford, CA). More results will be given at the 39th Denver X-ray conference (July 30-A ug. 3, 1990, Steamboat Springs, CO), the proceedings of which will be published. Finally, Dr. Gruner will report on these, and other CCD detector results during an invited address at the 48th Pittsburgh Diffraction Conference (October 31November 2, 1990, Huntsville, AL). Dr. Tate, the staff member supported under this grant, and Professor E. Eikenverry, of the Robert Wood Johnson Medical School, Piscataway, NJ, who was supported by this grant last year during his Sabbatical at Princeton University, have been the lead scientists on this project.

B) Assembly of a CCD-based Detector Utilizing a Fiber Optic Taper

Progress has also been made toward the assembly of a CCD detector based on a single-stage proximity focussed intensifier, a fiber optic reducing cone, and a Thomson 512 $\times 512$ CCD. As opposed to the device described above, which utilized a loaned, experimental CCD, and was primarily a test of feasibility, the Thomson CCD based detector will utilize more standard and readily available commercial components and will be suitable for rotating-anode $\mathrm{x}$-ray applications. This detector is being constructed as a permanent detector primarily for applicztion to in-house research problems at Princeton. It is expected that it will also be the basis for systems which would be commercially viable with presently available technology. Purchase of the components required for this device was made possible by support from the NSF, as a supplement to the basic detector research provided by the DoE. Neither source of support is individually adequate to cover the costs of fabricating the detector. 
Work to date has consisted of design of the detector and evaluation of components as received from vendors. Many years of experience in the construction of electro-optically based $x$-ray detectors have taught us that the key to successful detector design is very careful attention to the selection of the components. The philosopy being pursued with this device is to assemble a detector using components which are just at the leading edge of what is available commercially. As a result, each of the components are being ordered subject to specifications, which, in almost all cases, are beyond the off-the-shelf specifications, but not so much so that the vendors demand best-effort contracts. To date, the fiber optic tapers, low actinide fiber optic plates, the CCD controller, and most of the computer hardware have been delivered. The CCD and the intensifier are scheduled for delivery later this summer. The fiber optics have been evaluated and meet our specifications. The CCD controller was, in fact, the unit used to operate the large CCD described in the previous section, so it has now been field tested. (A new board has yet to be built to run the Thomson CCD, but this is a relatively small change to the CCD controller). Considerable work has also been performed on techniques of phosphor fabrication and on software development (see below) which will be required by the detector. The cryostat which will house the detector components is presently being machined. Our hope is to assemble the device for initial application trials by the end of this calendar year.

\section{C) New Phosphor Structures}

Phosphor development is crucial for efficient x-ray detection (Gruner et al, 1982; Gruner, $1987 ; 1989 \mathrm{~b})$ since, in most electro-optical detectors, x-rays are converted to light via phosphors. This musi be done efficiently and with high spatial resolution if the detcctor is to have operate with a high detective quantum efficiency and be capable of resolving fine spatial features in the diffraction patterns. Efficient, high stopping power, high resolution 
phosphor structures suitable for $\mathrm{x}$-ray detector work require novel methods of phosphor selection, deposition and evaluation. Time-resolved diffraction work poses special problems because the most commonly used phosphors, and the most efficient, have severe after-glow and trapping problems. There is a critical need for new phosphors and phosphor methods. Unfortunately, with the demise of the U.S.-based television manufacturing industry, phosphor research in this country has essentially come to a halt. For all these reasons, we have feit it important to devote resources to phosphor research.

Considerable work has been done within the last year on evaluating available phosphors and in fabricating screens suitable for diffraction problems. Whereas, formerly, our phosphor screens were fabricated by commercial CRT vendors, we are now depositing our own screens. Our fabrication process results in screens which are more robust, about twice as a efficient, and have better than twice the resolution of the commercial screens. We have found that commercially available phosphors are suitable for our applications, but must be grain sized and otherwise physically treated for optimum performance. An important component of this work has been the development of procedures which allows rapid testing of the fabricated screens. The white $\mathrm{x}$-ray beams used for Laue diffraction (Moffet et al, 1984 ) pose special phosphor problems because it is necessary to efficiently detect both high and low energy $\mathrm{x}$-rays in the same screen without degraded spatial resolution. Work on phosphors suitable for special applications, such as Laue diffraction, is an on-going process in our laboratory. But it emphasized that, already, considerable improvements over the state of the art have been accomplished and are already being used on our detectors.

The phosphor research which has been performed over the last year will be submitted for publication, probably within the next 5 months. (The reason that the phosphor work is not being written up immediately is that the authors are busily involved in preparing other results for publication, as will be made clear by this Progress Report.) 


\section{D) Software Developments}

Considerable progress has occured on the development of new detector software and on codes for the analysis of small-angle $\mathrm{x}$-ray scattering (SAXS) data. Typical CCD detectors produce 0.5 Mbyte of information every time the detector is read out; the $2048 \times 2048$ CCD detector described above spewed out 8 Mbytes of data with each image. Software for handling this volume of data is required if the detectors are to be efficiently utilized for scientific applications. Software development at Princeton is an on-going and integral part of the process of detector development and diffraction research.

Within the last year efficient codes have been devised for correction of the spatial and intensity sensitivity distortions introduced by detector components. Previouly published distortion correction procedures typically require several minutes on a large computer to correct a $512 \times 512$ pixel image. The newly devised correction procedures can accomplish this task within 15 seconds on a small PC. Considerable work has been done on detect $r$ r operating system software. The emphasis in the new codes is on portability so that codes do not have to be rewritten every time a new generation of computers is used. It is noteworthy that the operating systems developed at Princeton effectively merge the detector operation, data acquisition and data analysis processes. This has enormous benefits in terms of the efficiency of performing research. It is also noteworthy that we consider software to be public domain material and make it available to other research laboratories without charge. Software sets have been provided to several other laboratories; there is a growing string of requests for updates.

The new correction procedures will be described in a publication to be written this year. 


\section{E) High Pressure Apparatus}

Pressures in the range of 0 to 1 kbar are known to affect many of the processes of living cells, including such bizarre effects as the reversal of anesthesia and intoxication of alcohois (Janoff \& Miller, 1982). However, there is no known, accepted mechanism whereby such moderate pressures affect cellular function. We have suggested that biological pressure effects occur via the anisotropic compressibility of biomembranes and consequent effects on membrane proteins (see Section IIIB, below). Toward this end, apparatus suitable for investigation of the effects of moderate pressures ( 0 to $3 \mathrm{kbar})$ have been designed and applied to the study of biomembranes and bior. embrane components.

Three different kinds of pressure apparatus have been constructed. The most challenging has been a high pressure diffraction cell with concommitant pressure control equipment for use with SAXS. Prèsently, only one other laboratory (Dr. M. Caffrey's lab at Ohio Siate University) has the capability to perform SAXS on biological membranes in the 0-3 kbar range. Results which have been obtained with our apparatus, described in the research accomplishments section IIID, below, are presently being prepared for publication. A task for the next budget period is automation of the high-pressure control apparatus so that pressure dependent phase diagrams of membrane materials may be more readily acquired.

A second high pressure apparatus has been assembled for optical scattering studies of lipid-water dispersions undergoing pressure-induced phase transistions. For 1. ons which are not well understood, lyotropic liquid crystals and membrane dispersions cause large changes in the scattering of light when approaching mesomorphic phase transitions. We would like to understand the physics of this process so that it can be used as a structural probe. Preliminary descriptions of the apparatus and initial results have been reported in the Senior thesis of John Cerne and the General Examination Experiment description 
of Marc Potters, both of which are available from the Physics Department at Princeton University. The optical scattering results are complicated and promising, but not yet sufficiently understood to be ready for formal publication. Additional experiments and modificiations of the apparatus are planned for the coming year.

A third high pressure apparatus has been assembled to test the feasibility of performing pressure jumps in order to examine time-resolved, pressure-jump induced biomembrane lipid phase transitions at synchrotron radiation sources. Pressure jumps of $0.5 \mathrm{kbar}$ in 25 msec have been achieved, and faster jumps, if required, appear feasible by straightworward reduc ion of dead volume within the apparatus. The time scales which have already been achieved are adequate for examination of mesomorphic transitions, where typical time scales are 10 to $250 \mathrm{msec}$ (Tate, 1987; Tate et al, 1987). The pressure jump apparatus has been decribed in the General Examination Experiment description of Paul Harper, who is now continuing with the group as a Ph.D thesis student. Scheduling of the performance of the phase-transition study at a synchrotron source will depend on the availability of beam-time and personnel resources.

F) Lyoluminescent Dosimetry

Experiments have been continued on the study of lyoluminescence. This is the term applied to the emission of light dissolution of certain organic and inorganic solids after they have been exposed to ionizirig radiation, such as x-ray, gamma rays, electrons, neutrons, and so forth. Not only is the phenomenon of interest in its own right but its potential as a dosimetric technology is evident. The work at Princeton is directed toward developing a reliable measurement for very low doses. Much of the literature describes result sibtained at very high doses - kilorads and higher. Our work is more concerned with detecting millirads. Results to date confirm that the light is due to chemiluminescent processes and that in the dissolution of certain inorganic phosphors hydrated electrons are important, 
acting on complexes formed in the solvent. In addition to continuing experiments, a brief review of the subject is being prepared as a technical report.

Previous reports of the group on studies of sonoluminescence have repeatedly called attention to the possible biological (health) effects of ultrasound techniques used in medical procedures. Reaction to these warnings has been slow and materializing but a recent series of 4 papers appearing in Physics in Medicine and Biology describing work done at the Addenbrooke's Hospital, Cambridge, England, have taken serious note of the affect.

\section{III) APPLICATIONS RESEARCH ACCOMPLISHMENTS}

\section{A) New X-ray Structural Methods}

Structural determination of biomembrane lipid dispersions typically are performed via the mass allocation method of Luzzatti (1968). This method is tedious in that it requires many samples for the determination of the structure of excess water specimens and is unable to probe the detailed density variations which are believed to be important in lipid phase stability (Gruner, 1989). For these reasons, new direct structural methods, which involve Fourier inversion of the lipid dispersion diffraction patterns, have been developed and applied to bilayer and nonbilayer lipid phases (Turner \& Gruner, 1989; 1990). The results demonstrate that density variations exist, as predicted (Kirk et al, 1984), within non-bilayer lipid mesomorphs and lend considerable support to the theory of biomem-

brane phase transitions which has been developed at Princeton (e.g. see Gruner, 1989, for a summary). It was also shown that oils and hydrophobes which are known to exert significant biological effects pack in highly anisotropic ways in nonbilayer phases. The significance of these results are two-fold: First, it suggests a mechanism for the relative partitioning of medically important lipiphilic molecules amongst membranes of different lipid compositions. This may help explain the biodistribution of drugs amongst different 
cellular compartments. Second, it supports hypothesis that certain biomembrane compositions lead to internal bilayer strains which may affect protein function (Gruner, 1985; 1989). This may turn out to be imporiant toward understanding the lipid compositions of biomembranes.

The structural methods will be reported in detail in the Ph.D thesis of David Turner, which is slated for deposition by the end of the summer, and in a publication to be drawn therefrom.

\section{B) A Novel Mechanism for General Anesthesia}

Identification of the mechanism of general anesthesia is an outstanding medical research problem which, despite intensive investigation, has defied resolution for over a hundred years. No existing thory of anesthesia has adequately explained three observations: (1) Anesthetics are molecularly diverse, running from complex steroids to the inert gas, xenon (which is an excellent general anesthetic). (2) Anesthesia is reversed upon application of moderate pressures ( 0 to 400 bar). (3) Homologous chain anesthetics, such as the n-alcohols, exhibit a cut-off in effectiveness beyond a chain length which depends on the type of molecule.

Our studies on biomembrane lipid phase behavior have led to a mechanism which may be able to explain each of these observations and provide a mechanism for general anesthetic action. The mechanism follows from the hypothesis that the membrane spontaneous curvature parameter, which has been shown to control lipid mesomorphic phase behavior, affects integral membrane protein function (Gruner, 1989). For simple physical-chemical reasons, this parameter is sensitive to lipophilic compounds, such as general anesthetics, in a manner which cuts off at certain chain lengths. Furthermore, the spontaneous curvature parameter is the most pressure sensitive membrane parameter known (Shyamsunder et al, 1989). In particular, we have shown that the effects of physiologically relevant pressures, 
reverse the effects of n-alcohols at physiologically relevant concentrations. The basic argument, then, is that anesthetics slew the spontaneous curvature parameter of biomembranes away from homeostatically regulated values and that this affects crucial membrane proteins, such as ion pumps, which are likely to be involved in nerve excitation. Pressure can reverse these effects. Since the anesthetics affect colligative properties of lipid layers, the general requirements for a molecule to be an anesthetic is lipophilicity to certain depths in lipid bilayars.

The proposed mechanism is just starting to be explored experimentally. The most difficult aspect of the experim its will be demonstrating the effects on membrane proteins, because, in general, membrane protein reconstitutions and assays are hard to perform. The experimental program evolving from this project alone is likely to require several years of work. Toward this end, we are engaging in collaborations with other laboratories on the more biological aspects of the problems. The response from the larger biological and medical community has been enthusiastic; 5 different laboratories have expressed interest. in collaborations on various aspects of this project.

The anesthetic mechanism was presented as an invited paper at the Molecular and Cellular Nechanisms of Alcohol and Anesthetics Conference, June 25-28, 1990, Calgary, Alberta, Canada (Gruner \& Shyamsinder, 1990). and will appear in the Annals of the Vew Vork Academy of Sciences.

C) Elastic Properties of Membranes

The Princeton Biophysics group has become well known for its pioneering work on explaining membrane lipid phase behavior in terms of lipid layer elasticity (e.g., see Gruner, 1989, for a summary). The theory of phase behavior which has evolved makes detailed predictions about lipid phase behavior and has important implications for biomembrane function. I: has stimulated, and survived, deiailed scrutiny by many other major research 
groups in the larger lipid and biomembrane community (for recent examples, see Epand et al, 1989; Siegel et al, 1989; Sjolund et al, 1987, 1989; Watnick et al, 1990; see alsc reviews by Lindblom \& Rilfors, 1989; Seddon, 1989). Continued development of the thecry, cald experimental investigations of lipid elasticity are important parts of our research program. A number of significant new developments have occured within the last year:

A membrane elastic parameter, 'nnown as the Gaussian curvature parameter, was described theoretically almost 20 years ago (Helfrich, 1973), but has eluded experimental measurement, largely because it is related to a topological invariant. We have succeeded, for the first time, in measuring this parameter for a biomembrane lipid system by utilizing the change in topology of membrane structure which occurs across mesomorphic phase boundries. The sign and magnitude of the parameter are consistent with the values needed for the stability of cubic phases in the phase diagram. A first report of the results was given at the American Physical Society meeting in Anaheim, CA (Gruner et al, 1990). Detailed results will appear in the Ph.D thesis of David Turner and in a publication dirawn therefrom.

Osmotic compression experiments on a common biomembrane lipid have been used to demsonstrate a novel re-entrant bilayer phase behavior and to measure the energy of transition (Gawrisch et al, 1990). The transition energy is surprisingly small and points toward the importance of hydrocarbon packing factors in maintaining the stability of bilayer structures. This study combined $x$-ray and NMR techniques and, in the process, demonstrated novel NMR methods of probing lipid phase behavior. A manuscript describing the results is nearing final form and should be submitted to Biochemistry within the next two months. 
In another study combined deuterium NMR and $\mathrm{x}$-ray diffraction experiments were also used to investigate correlations between mesomorphically competent lipid compositions and the lipid hydrocarbon order parameter profile. The results confirmed that compositions anc temperatures just short of bilayer to nonlamellar mesomorphic transitions involve elastic stresses in bilayers which are visible as order parameter changes. The significance of these results is that it supports the hypothesis that curvature strain in bilayers can affect membrane protein function. Results will be reported at the Vancouver Biophysics Congress later this summer. A publication fully describing the results is planned.

\section{D) Effects of Pressure on Membranes}

Moderate pressures (up to $1 \mathrm{kbar}$ ) are known to affect many cellular functions. The mechanism of moderate pressure effects on biological organisms have remained a mystery because cellular components, such as water, lipid, carbohydrates, and proteins, are all characterized by typical solid or liquid bulk compressibility coefficients, which is to say that they are practically imcompressible at moderate pressures. Studies of the effects of pressure on proteins and upon the gel-to-liquid crystalline lipid transition have typically required thousands of atmospheres of pressure for the observation of quantitatively substantial effects. We have shown (Shyamsunder et al, 1989) that the spontaneous membrane curvature is extraordinarily pressure sensitive and that large scale mesomorphic transitions occur, in many cases, with the application of less than 100 bar of pressure. Lipid mesomorphic transitions are, in fact, the most pressure sensitive lyotropic phase transitions known. If membrane protein function is modulated by homeostatically regulated biomembrane spontaneous curvature, as has been hypothesized (Gruner, 1985; 1989), then it necessarily follows that moderate pressures will affect protein function.

Research effort is being devoted to understanding the physics of the pressure sensitivity of the spontaneous curvature. Toward this end, high pressure SAXS apparatus, as 
described in section IIE, above, was built and applied to investigate the pressure-induced structural changes which occur in lipid systems. The high sensitivity of the spontaneous curvature to pressure follows not from the bulk lipid compressibility, which is small, but from a high relative rate of change of the anisotropic lipid layer compressibility, ie, the relative rate of contraction of projected headgroup and chain cross-sectional molecular areas. The spontaneous curvature couples directly to the relative rate, so small magnitude differences can correspond to a large relative rate. These results, which are some of the first structural studies of lipid mesomorphism under pressure, are being prepared for publication and will be an large part of the Ph.D thesis of Peter So. The results have been augmented by high pressure calorimetry data. Future work will concentrate on understanding why the anisotropic compressibilities are so different. We also hope to coristruct a high pressure dilatometer and, using the Clausius-Clapeyron relation, relate the effects of pressure to changes in the entropy of the lipids, especially of the hydrocarbon chains.

\section{E) Neutron Diffraction Studies}

Neutron diffraction data gathered at Brookhaven National Laboratory was analyzed to understand the distribution of deuterated decane within the hydrocarbon zones of nonlamellar lipid phases. This is one of the first neutron diffration structural studies of nonlamellar mesomorphism in biornembrane lipids. The results clearly show that the decane, as first predicted on theoretical grounds (Kirk et al, 1984; Kirk \& Gruner, 1985), preferentially partition into particular regions of the lipid hydrocarbon volume of inverted hexagonal phases. These results are consistent with NMR studies of alkane partitioning (Sjolund et al, 1987; 1989; Siegel et al, 1989) which were stimulated by our earlier work. The neutron results are powerful because they are direct and because they allow determination of the distribution of the alkane within the lattice. We hope to use the distribution function to deternine the free energy of transfer of alkane from one part of the lattice 
to another, so as to understand the magnitude of chain stress present in the nonlamellar phase. Analysis of the neutron data was made possible by the new structural methods mentioned in section IIIA, above. These important results will appear in the Ph.D thesis of David Turner and a publication drawn therefrom. (It should be obvious by now that David Turner's thesis is extraordinary.)

\section{F) Novel Lipid and Surfactant Systems}

A direct consequence of the DoE support enjoyed by the Princeton Biophysics group is that the laboratory has some of the world's best facilities for the investigation of the structure of lipid and surfactant lyotropism. An explicit function of the laboratory is to provide access to these facilities by other groups for the study of novel lipids and surfactants; we typically service several dozen requests a year for x-ray analysis. Our feeling is that we have an obligation to provide such service to the community, both because the laboratory is supported on public funds and also because it often leads to good science. Most importantly, experience has shown that such service is a healthy route toward collaborations with other groups which have the capability to synthesis or bio-isolate new molecules and materials. Such collaborations are very important to us because, being in a physics department, we do not generally maintain in-house facilities for organic synthesis or biological isolation of animal or bacterial chemicals.

It is worth noting that personnel and beam-time limitations prevent us from honoring all the requests we receive for access to the $x$-ray facility. A persistent concern is that the very tight fiscal research climate of the last few years has led to an erosion of umbrella grant support, in terms of constant dollars, and have forced us to seek more, but relatively smaller grants. These grants have each, in turn been trimmed by Gramm-Rudman cuts. The activity which has been most affected by the concommitantly necessary scaling back of the research effort is precisely the outside user service component because, by its nature, 
results of such service are not listed amongst the initially proposed specifir gaals of our research grants. If funding cutbacks continue, even fewer outside requests will be serviced. In the long run, this can be very detrimental to to overall goals of publically funded research.

Only brief mention will be made of several significant studies of novel lipids have occured within the last year; more detail may be found in the publications listed:

Lewis et al (1989) studied the effects of hydrocarbon chain variation on lipid mesomorphism of a large number of phosphatidylethanolamines (PEs) which, although synthetic, commonly occur in nature. It was found that the phase behavior, when plotted on a reduced temperature scale, was an almost universal function of the effective chain length, ie, the longest linear run of carbons down the chain. The primary effect of chain variations, such as side branches and unsaturation was simply to change the zero of the temperature scale by shifting the chain melting temperature. This enormously simplifies the task of understanding how chain variation affects lipid mesomorphic properties.

Mannock et al (1990) reported on the effects of chirality on the calorimetric and mesomorphic properties of an extensive series of synthetic glucopyransyl glycerols.

Lewis et al (1990) and Kaufman et al (1990) described modifications of the phase behavior of bacterial membrane lipid extracts in response to changes in growth conditions. Both studies indicated that the organisms responded by preserving a high proportion of membrane lipids which tend to promote nonlamellar phases.

Boni et al (1990) reports on the polymorphic behavior of the lipid-like surfactant alpha-tocopherol hemisuccinate. This material is being used as a biodegradeable liposomal drug delivery system.

Eikenberry et al (1990) reports on the phase behavior of mixtures of the lipids POPE and POPC. Mixtures of these lipids have become quite popular as matrices for protein 
reconstitutions. This was the first comprehensive report of the phase diagram of these lipids. A planned publication will provide a more detailed description.

\section{IV) LITERATURE CITED}

1. L.T. Boni, W.R. Perkins, S.R. Minchey, L.E. Bolcsak, S.M. Gruner, P.R. Cullis, M.J. Hope and A.S. Janoff (1990). Polymorphic phase behavior of alpha-tocopherol hernisuccinate. Chem. Phys. Lipids (in press).

2. H.W. Deckman and S.M. Gruner (1986). Format alterations in CCD based electrooptic x-ray detectors. Nuc. Instr. Meth. 246: 527-533.

3. E.F. Eikenberry, P.E. Harper, D.A. Hajduk and S.M. Gruner (1990). Phase behavior of the POPE-POPC system. Biophys. J. 57: 468a.

4. R.M. Epand, K.S. Robinson, M.E. Andres and R.F. Epand (1989). Dependence of the bilayer to hexagonal phase transition on ampiphile chain length. Biochem. 28: 9398-9402.

5. K. Gawrisch, V.A. Parsegian, R.P. Rand, N. Fuller, M.W. Tate, D. Hajduk and S.M. Gruner (1990). A hexagonal-lamellar double transition of dioleoylphosphatidylethanolamine (DOPE). Characterization under osmotic and thermal perturbation. Biophys. J. 57: $263 \mathrm{a}$.

6. S.M. Gruner, J.R. Milch and G.T. Reynolds (1982). Survey of two-dimensional electrooptical x-ray detectors. Nuc. Instr. Meth. 195: 287-297.

7. S.M. Gruner (1985). Curvature hypothesis: Does the intrinsic curvature determine biomembrane lipid composition. A role for non-bilayer lipids. Proc. Natl. Acad. Sci. (USA) 82: 3665-3669.

8. S.M. Gruner (1987). Time resolved x-ray diffraction of biological materials. Science 238: 305-311.

9. S.M. Gruner (1989). Stability of lyotropic phases with curved interfaces. J. Phys. Chem. 93: 7562-7570.

10. S.M. Gruner (1989b). CCD and vidicon x-ray detectors: Theory and practice. Rev. Sci. Instr. 60: 1545-1551.

11. S.M. Gruner and E. Shyamsunder (1990). Is the mechanism of general anesthesia related to lipid membrane spontaneous curvature? Annals N.Y. Acad. Sci. (in press).

12. S.M. Gruner, D.C. Turner, Z-G. Wang and S.A. Safran (1990). Measurement of the Gaussian curvature coefficient of a lipid monolayer. Bull. Amer. Phys. Soc. 35: 257.

13. W. Helfrich (1973). Elastic properties of lipid bilayers: Theory and possible experiments. Z. fur Naturforschung 28C: 693-703.

14. A.S. Janoff and K.W. Miller (1982). A critical assessment of the lipid theories of general anesthetic action, in Biological Membranes, Vol. 4, D. Chapman, ed. pp. 417-476 (Academic Press, NY, 1982).

15. A.E. Kaufman, H. Goldfine, O. Narayan and S.M. Gruner (1990). Physical studies on the membranes and lipids of plasmologen-deficient Megasphaera elsdenii. Chem. Phys. Lipids (in press). 
16. G.L. Kirk, S.M. Gruner and D.L. Stein (1984). A thermodynamic model of the lamel$\operatorname{lar}\left(L_{\alpha}\right)$ to inverse hexagonal $\left(H_{I I}\right)$ phase transition of lipid membrane-water systems. Biochem. 23: 1093-1102.

17. G.L. Kirk and S.M. Gruner (1985). Lyotropic effects of alkanes and headgroup composition on the $L_{\alpha}-H_{I I}$ lipid liquid crystal phase transition: hydrocarbon packing versus intrinsic curvature. J. de Physique 46: 761-769.

18. R.N.A.H. Lewis, D.A. Mannock, R.N. McElhaney, D.C. Turner and S.M. Gruner (1988). Structural parameters affecting the lamellar to reversed hexagonal transition temperatures of phosphatidylethanolamines and monogly cosyl glycerolipids. Biophys. J. 53: $120 \mathrm{a}$.

19. R.N.A.H. Lewis, D.A. Mannock, R.N. McElhaney, D.C. Turner and S.M. Gruner (1989). The effect of fatty acid acyl chain length and structure on the lamellar gel to liquid crystalline and lamellar to reversed hexagonal phase transitions of aqueous phosphatidylethanolamine dispersions. Biochem. 28: 541-548.

20. R.N.A.H. Lewis, A.W.B. Yue, R.N. McElhaney, D.C. Turner and S.M. Gruner (1990). Thermotropic characterization of the 2-0-acyl, polyprenyl- $\alpha$-D-gluco pyranoside isolated from palmitate-enriched Acholeplasma laidlawii B membranes. Biochim. Biophys. Acta (in press).

21. G. Lindblom and L. Rilfors (1989). Cubic phases and isotropic structures formed by membrane lipids - possible biological relevance. Biochim. Biophys. Acta 988: 221-256.

22. V. Luzzati (1968). x-ray diffraction studies of lipid-water systerns. In Biological Membranes, Vol. I, D. Chapman, ed. (Academic Press, NY, pp. 71-123).

23. D.A. Mannock, R.N.A.H. Lewis, R.N. McElhaney, D.C. Turner and S.M. Gruner (1990). The effect of chirality on the bilayer and non-bilayer phase transitions in di-dodecyl- $\beta$-D-glucopyranosyl glycerols. Biophys. J. 57: 267 a.

24. K. Moffat, D. Szebenyi ard D. Bilderback (1984). X-ray Laue diffraction from protein crystals. Science 223: $1423_{-1425 .}$

25. J.M. Seddon (1989). Structure of the inverted hexagonal $\left(H_{I I}\right)$ phase, and nonlamellar phase transitions of lipids. Biochim. Biophys. Acta 1031: 1-69.

26. E. Shyamsunder, P. Botos and S.M. Gruner (1989). X-ray diffraction study of the effects of pressure on bilayer to non-bilayer lipid membrane phase transition. J. Chem. Phys. 90: 1293-1295.

27. D.P. Siegel, J. Banschbach and P.L. Yeagle (1989). Stabilization of $H_{I I}$ phases by low levels of diglycerides and alkanes: An NMR, calormietric and x-ray diffraction study. Biochem. 28: 5010-5019.

28. M. Sjolund, G. Lindblom, L. Rilfors and G. Arvidson (1987). Hydrophobic molecules in lecithin-water systems. I. Formation of reversed hexagonal phases at high and low water contents. Biophys. J. 52: 145-154. 
29. M. Sjolund, L. Rilfors and G. Lindblom (1989). Reversed hexagonal phase formation in lecithin-alkane-water systems with different acyl chain unsaturtaion and alkane length. Biochem. 28: 1323-1329.

30. M.W. Tate (1987). Equilibrium and kinetic states of the $L_{\alpha}-H_{I I}$ transitions. PhD thesis, Princeton University, Princeton, NJ.

31. M.W. Tate, S.M. Gruner, E. Shyamsunder and K.L. D'Amico" (1987). Kinetics of the lamellar-hexagonal phase transition in phospholipid water systems. Bull. Amer. Phys. Soc. 32: 843.

32. D.C. Turner and S.M. Gruner (1989). Electron density reconstruction of the inverted hexagonal $\left(H_{I I}\right)$ phase in phospholipid-water membranes. Biophys. J. 55: 116a.

33. D.C. Turner and S.M. Gruner (1990). Deviation from cylindrical symmetry in the inverted hexagonal $\left(H_{I I}\right)$ phase in phospholipid-water nembranes. Biophys. J. 57: $269 \mathrm{a}$.

34. P.I. Watnick, S.I. Chan and P. Dea (1990). Hydrophobic mismatch in gramacidin $A^{\prime} /$ lecithin systems. Biochem. 29: 6215-6221.

\section{V) PUBLICATIONS SINCE THE LAST PROGRESS REPORT}

\section{Papers}

1. R.P. Rand, N.L. Fuller, S.M. Gruner and V.A. Parsegian (1990). Membrane curvature, lipid segregation, and structural transitions for phospholipids under dual solvent stress. Biochem. 29: 76-87.

2. R.N.A.H. Lewis, D.A. Mannock, R.N. McElhaney, D.C. Turner and S.M. Gruner (1989). The effect of fatty acid chain length and structure on the lamellar gel to liquid crystalline and lamellar to reversed hexagonal phase transitions of aqueous phosphatidylethanolamine dispersions. Biochem. 28: 541-548.

3. S.M. Gruner (1989). CCD and vidicon x-ray detectors: Theory and practice. Rev. Sci. Instr. 60: 1545-1551.

4. H.W. Deckman, K.L. D'Amico, J.H. Dunsmuir, B.P. Flannery and S.M. Gruner (1988). Microtomography detector design: It's not just resolution. To be presented at the Denver X-ray Conf. Advances in X-ray Analysis Series (in press).

5. S.M. Gruner (1989). Stability of lyotropic phases with curved interfaces. Invited feature article. J. Phys. Chem. 93: 7562-7570.

6. S.M. Gruner (1989). Hydrocarbon chain conformation in the $H_{I I}$ phase. Letter to the Editor (regarding an article by J. Scherer, Biophys. J. 55 (1989) 965-971. Biophys. J. 56: 1045 .

7. Z.N-C. Ha and S.M. Gruner (1989). Evaluation of a linear self-scanned photodiode array detectur for direct detection of $6-8 \mathrm{keV}$-rays. Analytical Instrumentation 18: 197-212.

8. S.M. Gruner. Nonlamellar Lipid phases. To be published in The Structure and Function of Cell Membranes, P.L. Yeagle, ed. (Telford Press, Caldwell, NJ). 
9. L.T. Boni, W.R. Perins, S.R. Minchey, L.E. Bolcsak, S.M Gruner, P.R. Cullis, M.J. Hope and A.S. Janoff (1990). Polymorphic phase behavior of alpha-tocopherol hernisuccinate. Chem. Phys. Lipids (in press).

10. H.W. Deckman, J.H. Dunsmuir and S.M. Gruner (1990). Microfabrication of cellular phosphors. J. Vac. Sci. B 7: 1832-1835.

11. A.E. Kaufman, H. Goldfine, O. Narayan and S.M. Gruner (1990). Physical studies on the membranes and lipids of plasmologen-deficient Megasphaera elsdenii. Chem. Phys, Lipids (in press).

12. R.N.A.H. Lewis, A.W.B. Yue, R.N. McElhaney, D.C. Turner and S.M. Gruner (1990). Thermotropic characterization of the 2-0-ocyl, polyprenyl- $\alpha$-D-glucopyranoside isolated from palmitate enriched Acholeplasma laidlawii $B$ membrane. Biochim. Biophys. Acta (in press).

13. S.M. Gruner and E. Shyamsunder (1990). Is the mechanism of general anesthesia related to lipid membrane spontaneous curvature? Invited paper presented as "Mnlecular and Cellular Mechanisms of Alcohol and Anesthetics", University of Calgary, Alberta, Canadla, June 25-28, 1990. To appear in Annals of the New York Academy of Sciences.

Abstracts

1. D.C. Turner and S.M. Gruner, (1990). Deviation from cylindrical symmetry in the inverted hexagonal $\left(H_{I I}\right)$ phase in phospholipid-water membranes. Biophys. J. 57: 269 a.

2. P.T.C. So, M.W. Tate, S.M. Gruner and E. Shyamsunder (1990). X-ray diffraction studies on the effect of alkanols on DOPE-membranes under high pressure. Biophys. J. 57: $274 \mathrm{a}$.

3. E.F. Eikenberry, P.E. Harper, D.A. Hajduk and S.M. Gruner (1990). Phase behavior of the POPE-POPC system. Biophys. J. 57: 468a.

4. S.M. Gruner (1990). Relations between curvature elasticity, nonlamsllar phases, and biomembrane function. Biophys. J. 57: 20a.

5. K.Gawrisch, V.A. Parsegian, R.P. Rand, N. Fuller, M.W. Tate, D.Hajduk and S.M. Gruner (1990). A hexagonal-lamellar-hexagonal double transition of dioleylphosphatidylethanolamine (DOPE). Characterization under osmotic and thermal perturbation. Biophys. J. 57: 35a.

6. L.T. Boni, W.R. Perkins, S.R. Minchey, L.E. Bulczak, S.M. Gruner, P.R. Cullis, M.J. Hope and A.S. Janoff (1990). The polymorphic phase behavior of alpha-tocopherol hemisuccinate. Biophys. J. 57: 263a.

7. D.A. Mannock, R.N.A.H. Lewis, R.M. McElhaney, D.C. Turner ai A S.M. Gruner (1990). The effect of chirality on the bilayer and non-bilayer phase transitions in di-dodecyl- $\beta$-D-glucopyramosyl glycerols. Biophys. J. 57: 267a.

8. S.M. Gruner, D.C. Turner, Z-G. Wang and S.A. Safran (1990). Measurement of the Gaussian curvature coefficient of a lipid monolayer. Bull. Amer. Phys. Soc. 35: 257. 

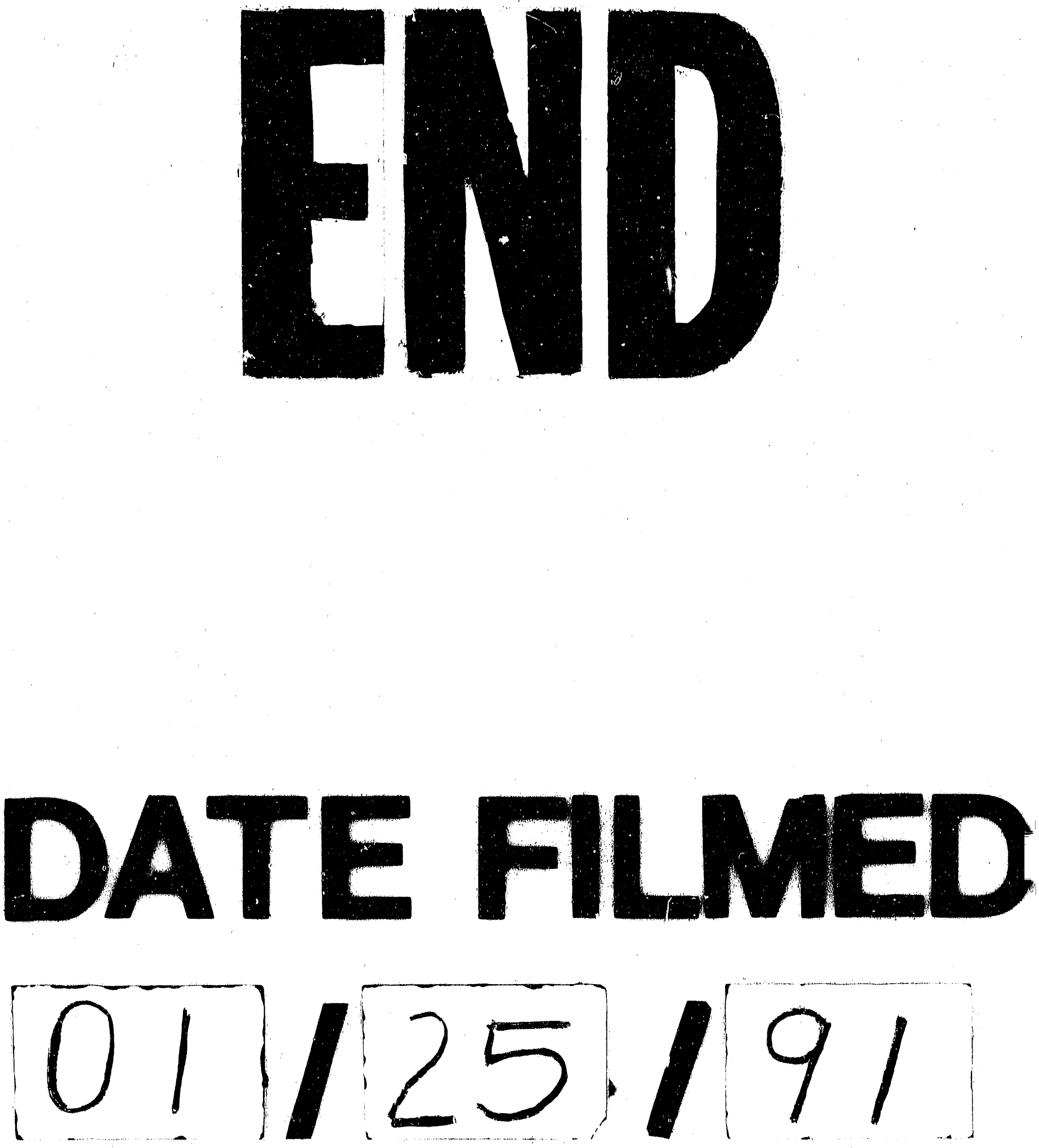
